Original Research Paper

\title{
Fast Approach to Factorize Odd Integers with Special Divisors
}

\author{
1,2,3Xingbo Wang and ${ }^{1} \mathrm{Junjian}$ Zhong \\ ${ }^{1}$ Department of Mechatronic Engineering, Foshan University, Foshan City, PRC, 528000, China \\ ${ }^{2}$ State Key Laboratory of Information Security, \\ Institute of Information Engineering, Chinese Academy of Sciences, Beijing 100093, China \\ ${ }^{3}$ Guangdong Engineering Center of Information Security for Intelligent Manufacturing System, China
}

Article history

Received: 04-12-2019

Revised: 13-01-2020

Accepted: 25-01-2020

Corresponding Authors:

Xingbo Wang

Department of Mechatronic

Engineering, Foshan University,

Foshan City, PRC, 528000,

China

Email: 153668@qq.com

\section{Introduction}

A Valuated Binary tree is a full perfect binary tree that has odd integers bigger than 1 put on it from top to bottom and left to right, as introduced in Wang's (2016a). With the help of the valuated binary tree, many new properties of the odd integers are discovered. For example, the properties of symmetric nodes and symmetric common divisors, the properties of subtree duplication and subtree transition and the properties of sum by level, root division and uniform sum were discovered in (Wang, 2016b; 2017a), the genetic properties of odd integers was disclosed in (Wang, 2017b) and the periodical divisibility traits along the leftmost path or the left side-path of the tree were demonstrated in (Wang and Guo, 2019). All these new properties enable us to know the integers in a different point of view, as stated and investigated in Wang's (2018). Integer factorization has been a hard problem in number theory and in cryptography over years, as overviewed in Yan's (2013), Sarnaik's et al. (2016) and Phulachand's (2016). Any new approach related with the integers shall of course be tried on the issue. Wang (2017b) proved that there should exist an algorithm of $O\left(\log _{2} N\right)$ searching steps to factorize an odd integer $N$. But there has not been a convincible demonstration. Thereby, this paper, continues the studies on integer factorization and proves that there are odd integers that can be factorized in $O\left(\log _{2} N\right)$ searching steps or in $O\left(\left(\log _{2} N\right)^{4}\right)$ bit operations.

\section{Preliminaries}

\section{Definitions and Notations}

A valuated binary tree $T$ is such a binary tree that each of its nodes is assigned a value. An odd number $N$ rooted tree, denoted by $T_{N}$ is a recursively constructed valuated binary tree whose root is the odd number $N$ with $2 N-1$ and $2 N+1$ being the root's left and right sons, respectively. Each son is connected with its father via a path, but there is no path between the two sons. $T_{3}$ tree is the case $N=3$. For convenience, symbol $N_{(k, j)}$ is by default the node at position $j$ on level $k$ of $T_{3}$, where $k=$ $1,2, \cdots$ and $j=0,1, \cdots, 2^{k}-1$. Symbol $N_{(k, j)}^{N}$ is to denote the node at position $j$ on level $k$ of $T_{N}$, where $k=1,2, \cdots$ and $j$ $=0,1, \cdots, 2^{k}-1$. Symbol $X \in l\left(T_{N}\right)$ means node $X$ is in the left branch of $T_{N}$ while symbol $X \in r\left(T_{N}\right)$ means $X$ is in the right branch of $T_{N}$. Symbol $N_{(i, 0)}^{N}$ is the leftmost node on level $i$ of $T_{N}$; use symbol $N_{(i,-1)}^{N}$ to denote the odd number left to $N_{(i, 0)}^{N}$, namely, $N_{(i,-1)}^{N}=N_{(i, 0)}^{N}-2$. Use symbol $P_{0}^{N}$ to indicate the leftmost path defined by $P_{0}^{N}=\left\{N_{(0,0)}^{N}, N_{(1,0)}^{N}, \cdots, N_{(i, 0)}^{N}, \cdots\right\}$ and symbol $P_{L}^{N}$ to indicate the path defined by $P_{L}^{N}=\left\{N_{(1,-1)}, \cdots, N_{(i,-1)}, \cdots, \ldots\right\}$, which is also called a left side-path, as depicted in Fig. 1. The leftmost path and the rightmost path together with their side-paths respectively are in all called border-path or simply border. 


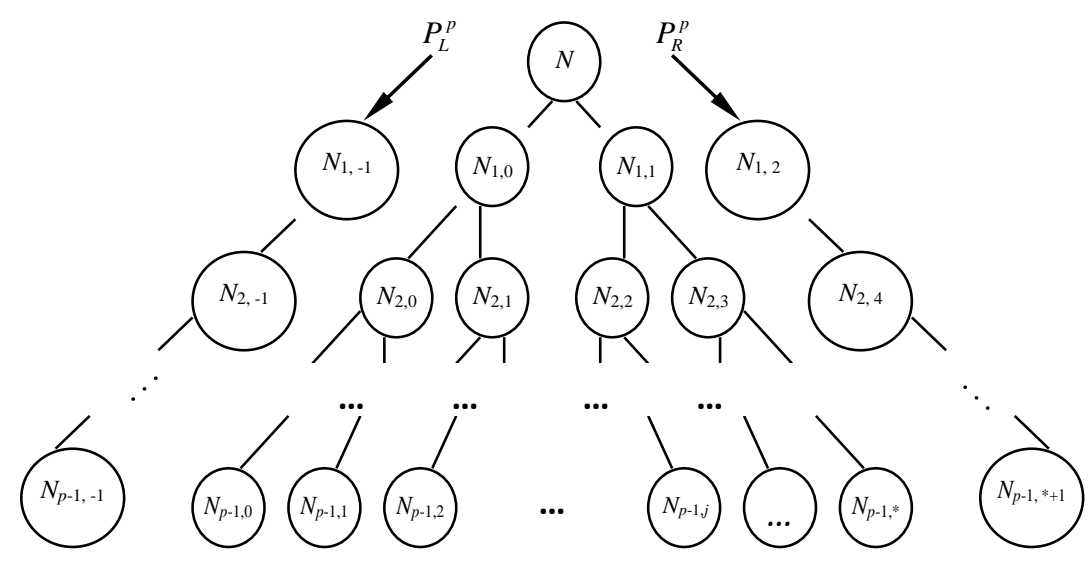

Fig. 1: $T_{p}$ Tree and its side-paths

Symbol $A \Rightarrow B$ means result $B$ is derived from condition $A$ or $A$ can derive $B$ out. In this whole article, symbol $\lfloor x\rfloor$ denotes the floor function, an integer function of the real number $x$ such that $x-1<\lfloor x\rfloor \leq x$ or equivalently $\lfloor x\rfloor \leq x<\lfloor x\rfloor+1$. Symbol $a \mid b$ means $b$ can be divided by $a$; symbol $(a, b)$ is to express the Greatest Common Divisor (GCD) of integers $a$ and $b$. A tracing step or a searching step is the computation of a father based on a son or vice versa.

\section{Lemmas}

Lemma 1 (Node Calculation, see in (Wang, 2016a))

Node $N_{(k, j)}$ of $T_{3}$ is calculated by:

$$
\begin{aligned}
& N_{(k, j)}=2^{k+1}+1+2 j \\
& k=0,1,2, \ldots ; j=0,1, \ldots, 2^{k}-1
\end{aligned}
$$

Node $N_{(k, j)}^{X}$ of $T_{X}$ is computed by:

$$
\begin{aligned}
& N_{(k, j)}^{X}=2^{k} X-2^{k}+2 j+1 \\
& k=0,1,2, \ldots ; j=0,1, \ldots, 2^{k}-1
\end{aligned}
$$

Lemma 2 (Divisors on Borders, see in (Wang and Guo, 2019))

Let $p$ be an odd integer and $T_{p}$ be the $p$-rooted valuated binary tree and $d$ be a positive integer with $1 \leq$ $d \leq p-1$; if there exits a positive integer $e$ such that $1 \leq e$ $\leq 2^{d-1}-1$ and $2^{d}-\underbrace{(2 e-1)}_{\text {odd }} \equiv 0(\bmod p)$, then $p \mid N_{(d, e-1)}^{p}$; if there exits a positive integer $f$ such that $0 \leq p \leq f \leq 2^{d-1}-2$ and $2^{d}+\underbrace{(2 f-1)}_{\text {odd }} \equiv 0(\bmod p)$, then $p \mid N_{(d, p-f)}^{p}$. Particularly, if $2^{d}-1 \equiv 0(\bmod p)$ then $N_{(d, 0)}^{p} \equiv 0(\bmod p)$; if $2^{d}+1 \equiv 0(\bmod p)$ then $N_{(d, p-1)}^{p} \equiv N_{(d,-1)}^{p} \equiv 0(\bmod p)$.
Lemma 3 (Floor Function, see in (Wang, 2019))

Properties of the floor functions with real numbers $x$ and $y$ and integers $n$ :

(P1) $\lfloor x\rfloor+\lfloor y\rfloor \leq\lfloor x+y\rfloor \leq\lfloor x\rfloor+\lfloor y\rfloor+1$

(P2) $\lfloor x\rfloor-\lfloor y\rfloor-1 \leq\lfloor x+y\rfloor \leq\lfloor x\rfloor-\lfloor y\rfloor<\lfloor x\rfloor-\lfloor y\rfloor+1$

(P13) $x \leq y \Rightarrow\lfloor x\rfloor \leq\lfloor y\rfloor$

(P32) $n\lfloor x\rfloor \leq\lfloor n x\rfloor \leq n(\lfloor x\rfloor+1)-1$. Taking $n=2$ yields $2\lfloor x\rfloor \leq\lfloor 2 x\rfloor \leq 2\lfloor x\rfloor+1$

\section{Main Results and Proofs}

\section{Theorem 1}

Let $p>1$ be an odd integer and $\alpha$ be a positive integer; if $p<2^{\alpha}+1$ then it holds:

$$
\begin{aligned}
& 2^{\alpha-1}<2^{\alpha-1}+\frac{p-1}{2} \leq 2^{\alpha}-1 \\
& 0 \leq 2^{\alpha-1}-\frac{p+1}{2} \leq 2^{\alpha-1}-1
\end{aligned}
$$

whereas if $p<2^{\alpha}-1$ it holds:

$$
\begin{aligned}
& 2^{\alpha-1}<2^{\alpha-1}+\frac{p-1}{2}<2^{\alpha}-1 \\
& 0<2^{\alpha-1}-\frac{p+1}{2} \leq 2^{\alpha-1}-1
\end{aligned}
$$

\section{Proof}

See the following deductions:

$$
\begin{aligned}
& p<2^{\alpha}+1 \Rightarrow \frac{p-1}{2}<2^{\alpha-1} \\
& \Rightarrow 2^{\alpha-1}<2^{\alpha-1}+\frac{p-1}{2}<2^{\alpha} \\
& \Rightarrow 2^{\alpha-1}<2^{\alpha-1}+\frac{p-1}{2} \leq 2^{\alpha}-1
\end{aligned}
$$




$$
\begin{aligned}
& p<2^{\alpha}+1 \Rightarrow \frac{p+1}{2}<2^{\alpha-1}+1 \\
& \Rightarrow-1<2^{\alpha-1}-\frac{p+1}{2}<2^{\alpha-1}-2 \\
& \Rightarrow 0 \leq 2^{\alpha-1}+\frac{p-1}{2} \leq 2^{\alpha}-1 \\
& p<2^{\alpha}-1 \Rightarrow \frac{p-1}{2}<2^{\alpha-1}-1 \\
& \Rightarrow 2^{\alpha-1}<2^{\alpha-1}+\frac{p-1}{2}<2^{\alpha}-1 \\
& \Rightarrow 2^{\alpha-1}<2^{\alpha-1}-\frac{p+1}{2}<2^{\alpha}-1 \\
& p<2^{\alpha}-1 \Rightarrow \frac{p+1}{2}<2^{\alpha-1} \\
& \Rightarrow 0<2^{\alpha-1}-\frac{p+1}{2}<2^{\alpha-1}-2 \\
& \Rightarrow 0<2^{\alpha-1}-\frac{p+1}{2} \leq 2^{\alpha-1}-1
\end{aligned}
$$

\section{Theorem 2}

Let $p>1$ be an odd integer and $\alpha$ be a positive integer; if $2^{\alpha}+1<p \leq 2^{\alpha+1}-1$ then it holds:

$$
2^{\alpha-1} \leq 2^{\alpha}+2^{\alpha-1}-\frac{p+1}{2}<2^{\alpha}-1
$$

and:

$$
0<2^{\alpha-1}-2^{\alpha}+\frac{p-1}{2} \leq 2^{\alpha-1}-1
$$

\section{Proof}

See the following deductions:

$$
\begin{aligned}
& 2^{\alpha}+1<p \leq 2^{\alpha-1}-1 \\
& \Rightarrow 2^{\alpha}+2<p+1 \leq 2^{\alpha+1} \\
& \Rightarrow 2^{\alpha-1}+1<\frac{p+1}{2} \leq 2^{\alpha} \\
& \Rightarrow-2^{\alpha} \leq-\frac{p+1}{2}<-2^{\alpha-1}-1 \\
& \Rightarrow \underbrace{2^{\alpha}+2^{\alpha-1}}_{\text {added items }}-2^{\alpha} \leq \underbrace{2_{\text {item }}^{\alpha}}_{\text {added } 2_{\text {items }}^{\alpha}+2^{\alpha-1}}-\frac{p+1}{2} \\
& <\underbrace{2^{\alpha}+2^{\alpha-1}-2^{\alpha-1}-1}_{\text {added items }} \\
& \Rightarrow 2^{\alpha-1} \leq 2^{\alpha}+2^{\alpha-1}-\frac{p+1}{2}<2^{\alpha}-1
\end{aligned}
$$

$$
\begin{aligned}
& 2^{\alpha}+1<p \leq 2^{\alpha+1}-1 \\
& \Rightarrow 2^{\alpha}<p-1 \leq 2^{\alpha+1}-2 \\
& \Rightarrow 2^{\alpha-1}<\frac{p-1}{2} \leq 2^{\alpha}-1 \\
& \Rightarrow \underbrace{2^{\alpha-1}-2^{\alpha}}_{\text {added items }}+2^{\alpha-1}<\underbrace{2^{\alpha-1}-2^{\alpha}}_{\text {added items }}+\frac{p-1}{2} \\
& \leq \underbrace{2^{\alpha-1}+2^{\alpha}}_{\text {added items }}+2^{\alpha}-1 \\
& \Rightarrow 0<2^{\alpha-1}-2^{\alpha}+\frac{p-1}{2}<2^{\alpha-1}-1
\end{aligned}
$$

\section{Theorem 3}

Let $N=p q$ with $1<p \leq q$ being odd integers; then $\left\lfloor\log _{2} N\right\rfloor \geq \max \left(2\left\lfloor\log _{2} p\right\rfloor,\left\lfloor\log _{2} q\right\rfloor\right)$.

Proof

Without loss of generality, assume $1<p \leq \sqrt{N} \leq q$. Then By Lemma 3 (P13) and (P32):

$$
\log _{2} N>\log _{2} q \Rightarrow\left\lfloor\log _{2} N\right\rfloor \geq\left\lfloor\log _{2} q\right\rfloor
$$

and:

$$
\log _{2} N \geq 2 \log _{2} p \Rightarrow\left\lfloor\log _{2} N\right\rfloor \geq\left\lfloor 2 \log _{2} p\right\rfloor \geq 2\left\lfloor\log _{2} p\right\rfloor
$$

Hence it holds:

$$
\left\lfloor\log _{2} N\right\rfloor \geq \max \left(2\left\lfloor\log _{2} p\right\rfloor,\left\lfloor\log _{2} q\right\rfloor\right)
$$

\section{Corollary 1}

Suppose $p$ and $q$ are odd integers with $1<p<q$; then $N=p q$ can be factorized in $\left\lfloor\log _{2} N\right\rfloor+1$ searching steps if one of $p$ and $q$ is in the form $2^{\alpha}+1$ or $2^{\alpha}-1$ with $\alpha$ being a positive integer.

\section{Proof}

According to the given conditions, there are 4 cases, $q=2^{\alpha}+1, q=2^{\alpha}-1, p=2^{\alpha}+1$ and $p=2^{\alpha}-1$, to be considered.

Consider the first case $q=2^{\alpha}+1$; then $N=2^{\alpha} p+p$. Rewrite this by:

$$
N=2^{\alpha} p-2^{\alpha}+2^{\alpha}+p=2^{\alpha} p-2^{\alpha}+2\left(2^{\alpha-1}+\frac{p-1}{2}\right)+1
$$

Referring to Lemma 1 and Theorem 1, it yields:

$$
N=N_{\left(\alpha, 2^{\alpha-1}+\frac{p-1}{2}\right)}^{p}
$$


This implies that $N$ is a node in the right branch of $T_{p}$. Consequently, there are at most $\alpha$ steps by tracing upwards and finding out the GCD between $N$ and its ancestors in $T_{p}$. Since $q=2^{\alpha}+1$, it yields:

$\alpha=\left\lfloor\log _{2}(q-1)\right\rfloor \leq \log _{2} q<\left\lfloor\log _{2} q\right\rfloor+1$

For the case $q=2^{\alpha}-1$, it holds $N=2^{\alpha} p-p=2^{\alpha} p-2^{\alpha}$ $+2\left(2^{\alpha-1}-\frac{p+1}{2}\right)+1$. Again referring to Theorem 1 , it leads to $N=N_{\left(\alpha, 2^{\alpha-1}-\frac{p+1}{2}\right)}^{p}$. This case says $N$ is a node in the left branch of $T_{p}$.

For the case $p=2^{\alpha}+1$ or $p=2^{\alpha}-1$, by Lemma 2, it knows $N_{(\alpha,-1)}^{p} \equiv 0(\bmod p)$ or $N_{(\alpha, 0)}^{p} \equiv 0(\bmod p)$ respectively. Since $\alpha \leq\left\lfloor\log _{2} p\right\rfloor+1$, by genetic property it knows $p$ can be found in at most $2\left\lfloor\log _{2} p\right\rfloor+1$ steps by tracing downwards and finding the GCD between $N$ and nodes along the leftmost path or left side-path of $T_{N}$.

\section{Example 1}

Let $N=527$; then $N$ 's ancestors are $263,131,65,33$ and 17, as depiected with Fig. 2. It can see that 17 is the divisor of $527=17 \times 31$ and $31=2^{5}-1$.

\section{Example 2}

Let $N=561$, then $N$ 's ancestors are $281,141,71,35$ and 17, as depiected with Fig. 3. It can see that 17 is the divisor of $561=17 \times 33$ and $33=2^{5}+1$.

\section{Proposition 1}

Suppose $p$ and $q$ are odd integers with $1<p<q$; then $N=p q$ can be factorized in $\left\lfloor\log _{2} N\right\rfloor+1$ searching steps if $q$ is in either form of $2^{\alpha}-1$ and $2^{\alpha}+1$ with $\alpha$ being a positive integer.

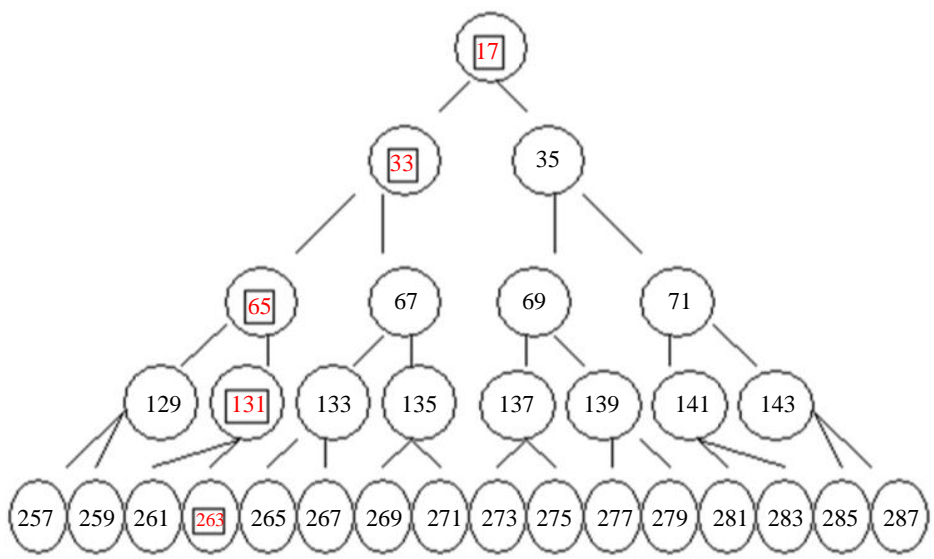

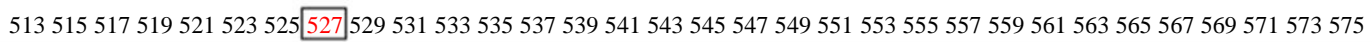

Fig. 2: The ancestors of $N=527$ in $T_{17}$

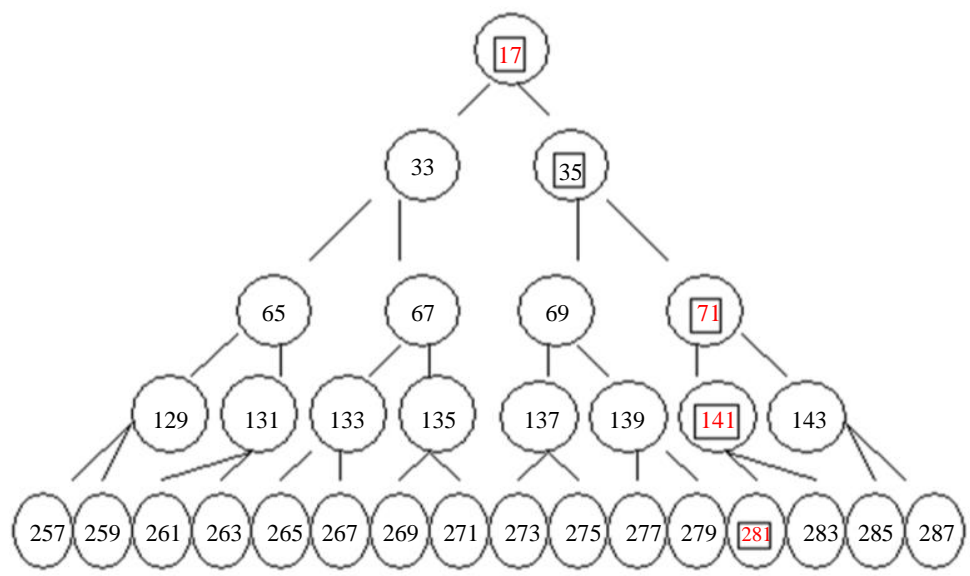

513515517519521523525527529531533535537539541543545547549551553555557559561563565567569571573575

Fig. 3: The ancestors of $N=561$ in $T_{17}$ 


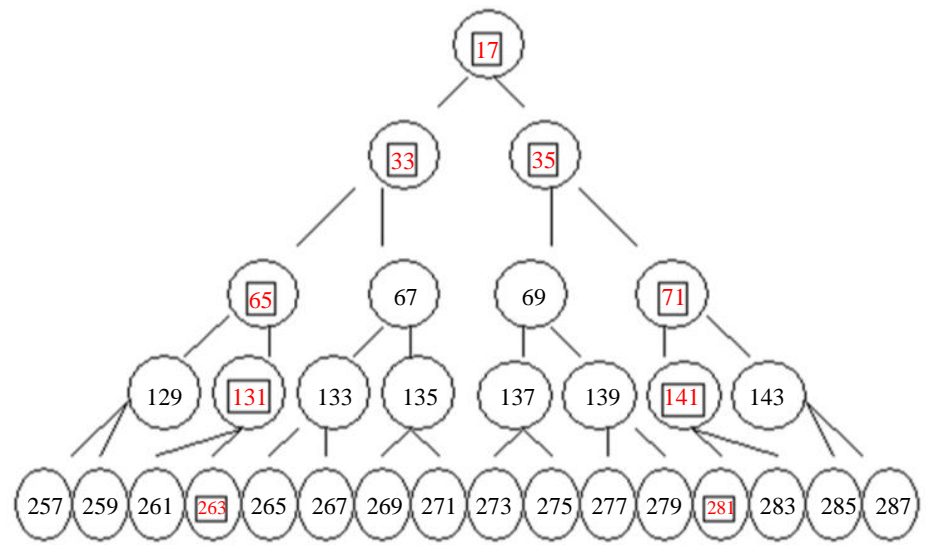

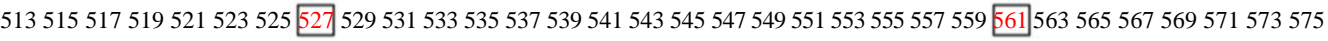

Fig. 4: Symmetric divisors with $q=31$ and $q=33$

\section{Example 3}

Figure 4, symmetric divisors distributed in a tree are again exhibited with $q$ in the form $2^{\alpha}-1$ or $2^{\alpha}+1$.

\section{Example 4}

Let $N=731$; then the left side-path of $T_{731}$ is 1459 , 2919, 5839 and 11679, as depicted in Fig. 5. It can see $\operatorname{GCD}(11679,731)=17$. Likewise, the right side path is $1465,2929,5857$ and 11713, among which it fits $\operatorname{GCD}(11713,731)=17$.

\section{Corollary 2}

Let $p$ and $q$ be odd integers with $1<p<q$ and suppose $q=2^{\alpha} u+1$ with $u \geq 1$ being an old integer, $\alpha$ being a positive integer and $1<p<2^{\alpha}+1$; then $N=p q$ can be factorized in $\left\lfloor\log _{2} p\right\rfloor+1$ searching steps.

\section{Proof}

The condition $q=2^{\alpha} u+1$ leads to:

$$
\begin{aligned}
& N=\left(2^{\alpha} u+1\right) p=2^{\alpha} u p-2^{\alpha}+2^{\alpha}+p \\
& =2^{\alpha} u p-2^{\alpha}+2\left(2^{\alpha-1}+\frac{p-1}{2}\right)+1
\end{aligned}
$$

Since $1<p<2^{\alpha}+1$, it knows by $1,2^{\alpha-1}<2^{\alpha-1}+\frac{p-1}{2}$ $\leq 2^{\alpha}-1$. Thereby:

$$
N=N_{\left(\alpha, 2^{\alpha-1}+\frac{p-1}{2}\right)}^{u p}
$$

This says that $N$ is a node in the right branch of $T_{u p}$. Thus there are at most $\alpha$ searching steps to trace upwards and find out the GCD between $N$ and its ancestors in $T_{u p}$.

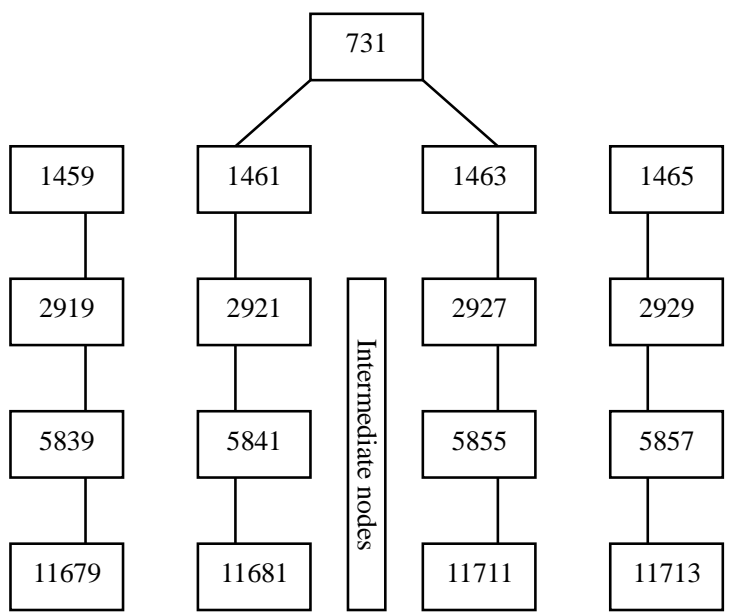

Fig. 5: Side-paths and border-path of $T_{731}$

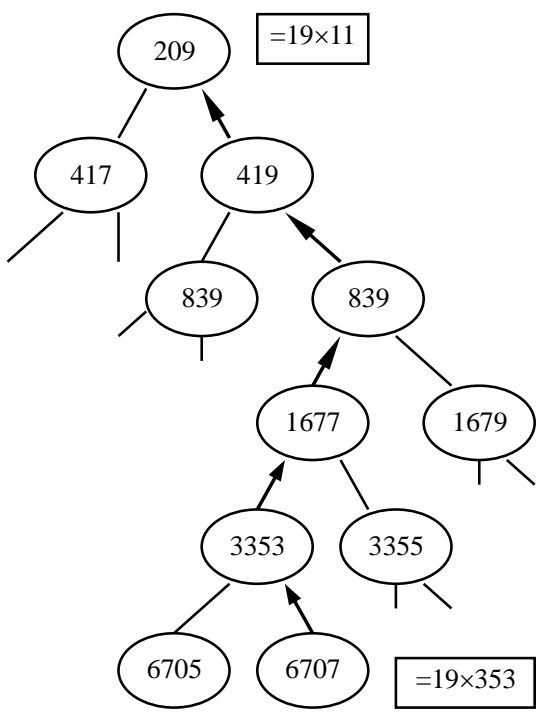

Fig. 6: Tracing ancestors of 6707 


\section{Example 5}

Let $N=6707$; then $N$ 's ancestors are 3353, 1677 , 839 , 419, 209, among which $\operatorname{GCD}(6707,209)=19$, which results in $6707=19 \times 353=19 \times\left(2^{5} \times 11+1\right)$. Figure 6 shows the tracing path from 6707 to 209. Seen from the figure, $N=6707$ is sure in the right branch of $\mathrm{T}_{209}$.

\section{Corollary 3}

Let $p$ and $q$ be odd integers with $1<p<q$ and suppose $q$ $=2^{\alpha} u-1$ with $u \geq 1$ being an old integer, $\alpha$ being a positive integer and $1<p<2^{\alpha}+1$; then $N=p q$ can be factorized in $\left\lfloor\log _{2} p\right\rfloor+1$ searching steps.

\section{Proof}

By Theorem 1, the condition $1<p<2^{\alpha}+1$ leads to $0 \leq$ $2^{\alpha-1}-\frac{p+1}{2} \leq 2^{\alpha-1}-1$. Considering:

$$
\begin{aligned}
& N=\left(2^{\alpha} u-1\right) p=2^{\alpha} u p-2^{\alpha}+2^{\alpha}-p \\
& =2^{\alpha} u p-2^{\alpha}+2\left(2^{\alpha-1}-\frac{p+1}{2}\right)+1
\end{aligned}
$$

it knows:

$$
N=N_{\left(\alpha, 2^{\alpha-1}-\frac{p+1}{2}\right)}^{u p}
$$

This says that $N$ is a node in the left branch of $T_{u p}$. Thus there are at most $\alpha$ searching steps to trace upwards and find out the GCD between $N$ and its ancestors in $T_{u p}$.

\section{Example 6}

Let $N=45601$; then $N$ 's ancestors are 22801, 11401, 5701, 2851, 1425, 713, among which $\operatorname{GCD}(45601,713)=31$, which results in $45601=$ $31 \times 1471=31 \times\left(2^{6} \times 23-1\right)$. Figure 7 shows the tracing path from 45601 to 713 . Seen from the figure, $N=$ 45601 is sure in the left branch of $T_{713}$.

\section{Corollary 4}

Let $p$ and $q$ be odd integers with $1<p<q$ and suppose $q=2^{\alpha} u+1$ with $u \geq 1$ being an old integer, $\alpha$ being a positive integer and $1<p<2^{\alpha}-1$; then $N=p q$ can be factorized in $\left\lfloor\log _{2} p\right\rfloor+1$ searching steps.

\section{Proof}

By Theorem 1, the condition $1<p<2^{\alpha}-1$ leads to $2^{\alpha-1}$ $<2^{\alpha-1}+\frac{p+1}{2}<2^{\alpha}-1$. Since:

$$
\begin{aligned}
& N=\left(2^{\alpha} u+1\right) p=2^{\alpha} u p-2^{\alpha}+2^{\alpha}+p \\
& =2^{\alpha} u p-2^{\alpha}+2\left(2^{\alpha-1}+\frac{p-1}{2}\right)+1 \\
& =N_{\left(\alpha, 2^{\alpha-1}+\frac{p-1}{2}\right)}^{u p}
\end{aligned}
$$

it knows that $N$ is a node in the right branch of $T_{u p}$. Thus there are at most $\alpha$ searching steps to trace upwards and find out the GCD between $N$ and its ancestors in $T_{u p}$.

\section{Example 7}

Let $N=42711$; then $N$ 's ancestors are 21355, 10677, 5339, 2669, 1335, 667, among which GCD(42711,667) $=23$, which results in $42711=23 \times 1857=$ $23 \times\left(2^{6} \times 29+1\right)$. Figure 8 shows the tracing path from 42711 to 667 . Seen from the figure, $N=42711$ is sure in the right branch of $T_{667}$.

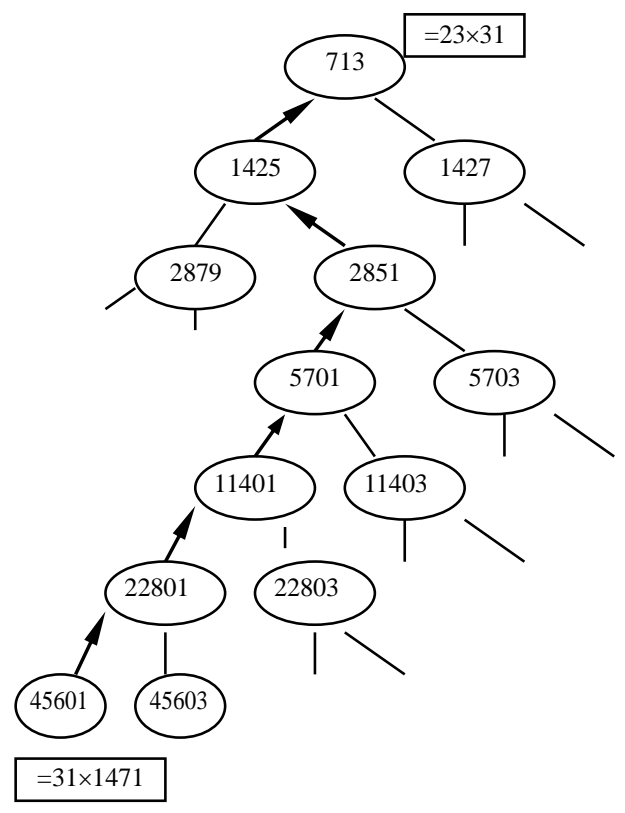

Fig. 7: Tracing ancestors of 45601

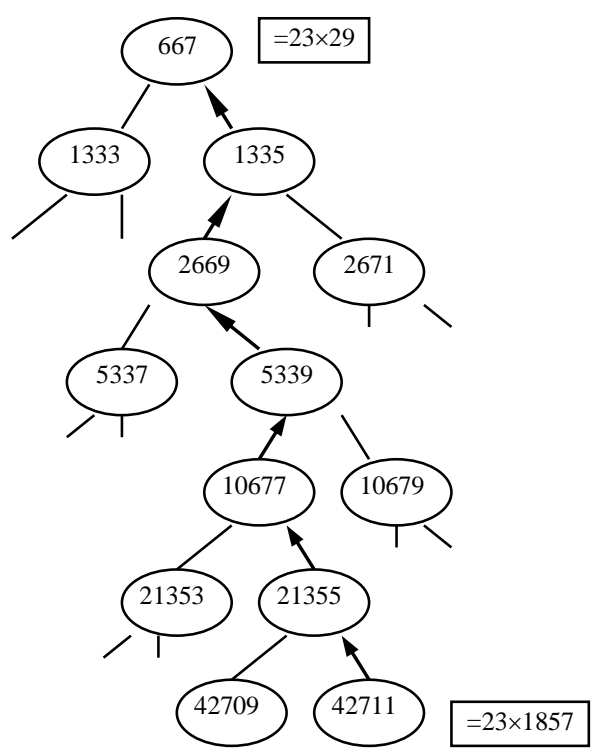

Fig. 8: Tracing ancestors of 42711 


\section{Corollary 5}

Let $p$ and $q$ be odd integers with $1<p<q$ and suppose $q=2^{\alpha} u$-1 with $u \geq 1$ being an old integer, $\alpha$ being an positive integer and $1<p<2^{\alpha}-1$; then $N=p q$ can be factorized in $\left\lfloor\log _{2} p\right\rfloor+1$ searching steps.

\section{Proof}

By Theorem 1, the condition $1<p<2^{\alpha}-1$ leads to 0 $<2^{\alpha-1}-\frac{p+1}{2} \leq 2^{\alpha-1}-1$. Since:

$$
\begin{aligned}
& N=\left(2^{\alpha} u-1\right) p=2^{\alpha} u p-2^{\alpha}+2^{\alpha}-p \\
& =2^{\alpha} u p-2^{\alpha}+2\left(2^{\alpha-1}-\frac{p+1}{2}\right)+1 \\
& \left.=N_{\left(\alpha, 2^{\alpha-1}\right.}^{u p}-\frac{p+1}{2}\right)
\end{aligned}
$$

it knows that $N$ is a node in the left branch of $T_{u p}$. Thus there are at most $\alpha$ searching steps to trace upwards and find out the GCD between $N$ and its ancestors in $T_{u p}$.

\section{Example 8}

Let $N=383031$; then $N$ 's ancestors are 191515, 95757, 47879, 23939, 11969, 5985 and 2993, among which $\operatorname{GCD}(383031,2993)=73$, which results in Figure 9 shows the tracing path from 383031 to 2993. Seen from the figure, $N=383031$ is sure in the left branch of $T_{2993}$.

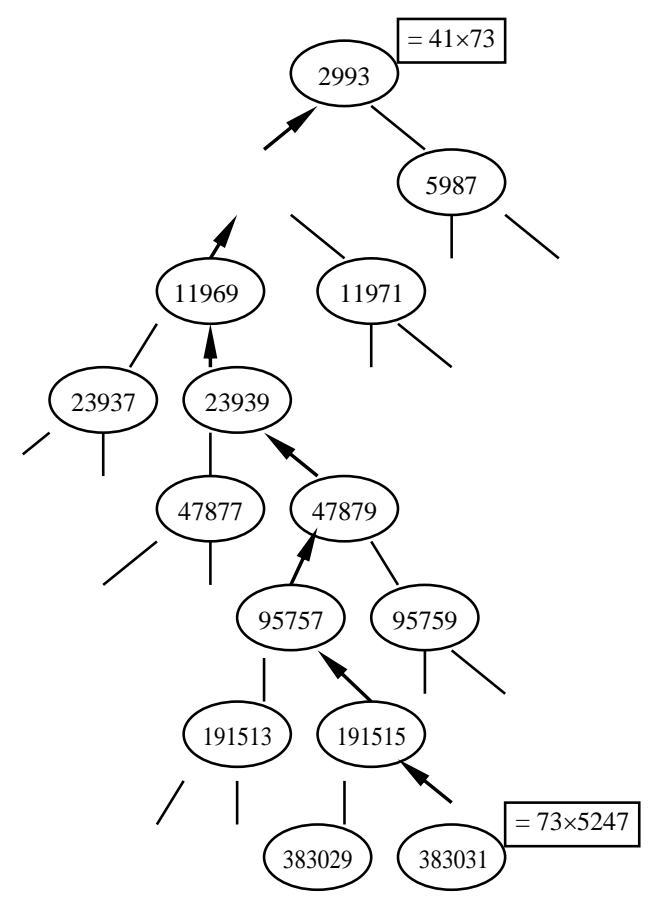

Fig. 9: Tracing ancestors of 383031

\section{Theorem 4}

Let $N=p q$ be an odd integer with $p$ and $q$ being odd integers and $1<p<q$; suppose $q=2^{\alpha} u \pm 1$ with $u \geq 1$ being an old integer, and $1<p \leq 2^{\alpha} \pm 1$; then $N$ can be factorized in $\left\lfloor\log _{2} N\right\rfloor+1$ steps or in $O\left(\left(\log _{2} N\right)^{4}\right)$ bit operations.

Proof

$$
\text { Let } J_{1}=2^{\alpha-1}-\frac{p+1}{2} \text { and } J_{2}=2^{\alpha-1}+\frac{p-1}{2} \text {; }
$$

summarizing Corollaries 1 to 5 yields Table 1 .

Seen from the table and referring to the Corollaries 1 to 5, it knows the theorem holds considering it needs $O\left(\left(\log _{2} N\right)^{4}\right)$ bit operations in computation of the GCD at each step.

\section{Corollary 6}

Let $N=p q$ be an odd integer with $p$ and $q$ being odd integers and $1<p<q$; suppose $q=2^{\alpha} u$-1 with $u \geq 1$ being an old integer and $\alpha$ being an positive integer; if $2^{\alpha}$ $+1<p \leq 2^{\alpha+1}-1$ then $N$ can be factorized in $\left\lfloor\log _{2} N\right\rfloor+1$ searching steps.

\section{Proof}

Direction calculation yields:

$$
\begin{aligned}
& N=\left(2^{\alpha} u-1\right) p=2^{\alpha} u p-p \\
& =2^{\alpha}(u p-2)-2^{\alpha}+2\left(2^{\alpha}+2^{\alpha-1}-\frac{p+1}{2}\right)+1 \\
& =2^{\alpha}((\underline{u p-2})-1)+2\left(2^{\alpha}+2^{\alpha-1}-\frac{p+1}{2}\right)+1
\end{aligned}
$$

Let $n=u p-2$; by Theorem $2,2^{\alpha-1} \leq 2^{\alpha}+2^{\alpha-1}-\frac{p+1}{2}<$ $2^{\alpha}-1$; consequently:

$$
N=N_{\left(\alpha, 2^{\alpha}+2^{\alpha-1}-\frac{p+1}{2}\right)}^{n}
$$

That is to say, tracing upwards from $N$ by $\alpha$ steps will reach $n$, the node left to $u p$; then:

$$
p=G C D(n+2, N)
$$

The relations described in Corollary 6 among $n, N$ and $u p$ are illustrated in Figure 10.

\section{Corollary 7}

Let $N=p q$ be an odd integer with $p$ and $q$ being odd integers and $1<p<q$; suppose $q=2^{\alpha} u+1$ with $u \geq 1$ being an old integer and $\alpha$ being an positive integer; if $2^{\alpha}$ $+1<p \leq 2^{\alpha+1}-1$ then $N$ can be factorized in $\left\lfloor\log _{2} N\right\rfloor+1$ searching steps. 
Table 1: Summarized cases from Corollaries 1 to 5

\begin{tabular}{|c|c|c|c|c|}
\hline$q$ & $p$ & $J$ & $N$ & \\
\hline \multirow[t]{2}{*}{$q=2^{\alpha} u-1$} & $1<p \leq 2^{\alpha}-1$ & $0<2^{\alpha-1}-\frac{p+1}{2} \leq 2^{\alpha-1}-1$ & $N=N_{\left(\alpha, 2^{\alpha-1}-\frac{p+1}{2}\right)}^{u p}$ & $N \in l\left(T_{u p}\right)$ \\
\hline & $1<p \leq 2^{\alpha}+1$ & $0 \leq 2^{\alpha-1}-\frac{p+1}{2} \leq 2^{\alpha-1}-1$ & & \\
\hline \multirow[t]{2}{*}{$q=2^{\alpha} u+1$} & $1<p \leq 2^{\alpha}-1$ & $2^{\alpha-1}<2^{\alpha-1}+\frac{p-1}{2}<2^{\alpha-1}-1$ & $N=N_{\left(\alpha, 2^{\alpha-1}+\frac{p-1}{2}\right)}^{u p}$ & $N \in r\left(T_{u p}\right)$ \\
\hline & $1<p \leq 2^{\alpha}+1$ & $2^{\alpha-1}<2^{\alpha-1}+\frac{p-1}{2} \leq 2^{\alpha-1}-1$ & & \\
\hline
\end{tabular}

\section{Proof}

Direction calculation yields:

$$
\begin{aligned}
& N=\left(2^{\alpha} u+1\right) p=2^{\alpha} u p+p \\
& =2^{\alpha} u p+2^{\alpha+1}-2^{\alpha}-2^{\alpha+1}+2^{\alpha}+p \\
& =2^{\alpha} u p+2^{\alpha+1}-2^{\alpha}+2\left(2^{\alpha-1}-2^{\alpha}+\frac{p-1}{2}\right)+1 \\
& =2^{\alpha}((\underline{u p+2})-1)+2\left(2^{\alpha-1}-2^{\alpha}+\frac{p-1}{2}\right)+1
\end{aligned}
$$

Let $n=u p+2$; by Theorem 2, $0<2^{\alpha-1}-2^{\alpha}+\frac{p-1}{2} \leq$ $2^{\alpha-1}-1$; consequently:

$$
N=N_{\left(\alpha, 2^{\alpha}+2^{\alpha-1}-\frac{p+1}{2}\right)}^{n}
$$

That is to say, tracing upwards from $N$ by $\alpha$ steps will reach $n$, the node left to $u p$; then:

$$
p=G C D(n-2, N)
$$

The relations described in Corollary 7 among $n, N$ and $u p$ are illustrated in Fig. 11.

\section{Theorem 5}

Let $N=p q$ be an odd integer with $p$ and $q$ being odd integers and $1<p<q$; suppose $q=2^{\alpha} u \pm 1$ with $u$ being an old integer and $\alpha$ being an positive integer; if $2^{\alpha}+1<$ $p \leq 2^{\alpha+1}-1$ then $N$ can be factorized in $3\left\lfloor\log _{2} N\right\rfloor+1$ searching steps or in $O\left(\left(\log _{2} N\right)^{4}\right)$ bit operations.

\section{Proof}

Summarizing Corollaries 6 and 7 yields to Table 2 .

Table 2 shows that, $N$ is a node of $T_{u p+2}$ or $T_{u p-2}$. Hence it easy to trace upwards from $N$ to $u p+2$ or $u p-2$ and then find out the divisor $p$. The time complexity is demonstrated in section 4.1.

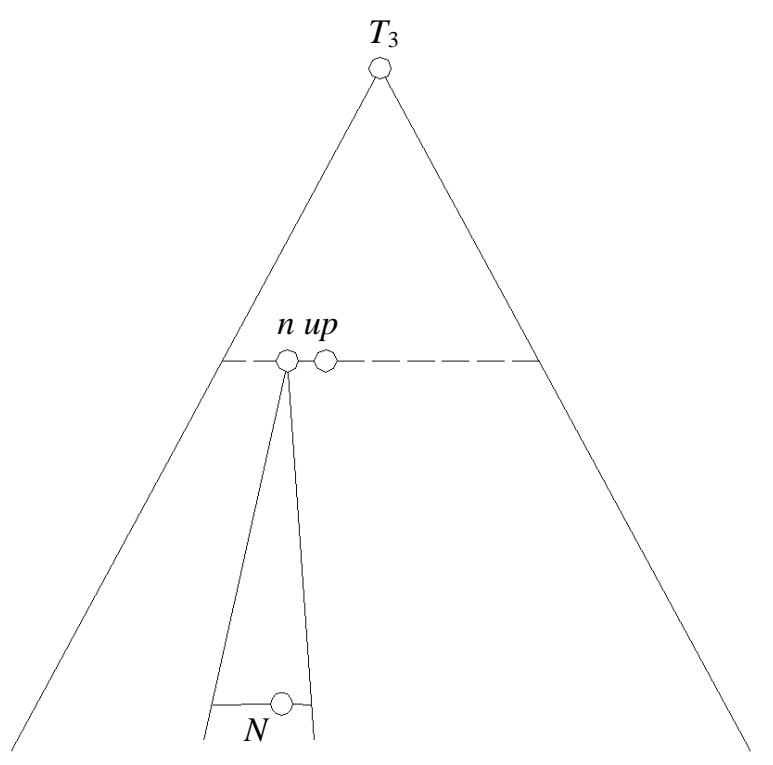

Fig. 10: Relations among $n, N$ and $u p$

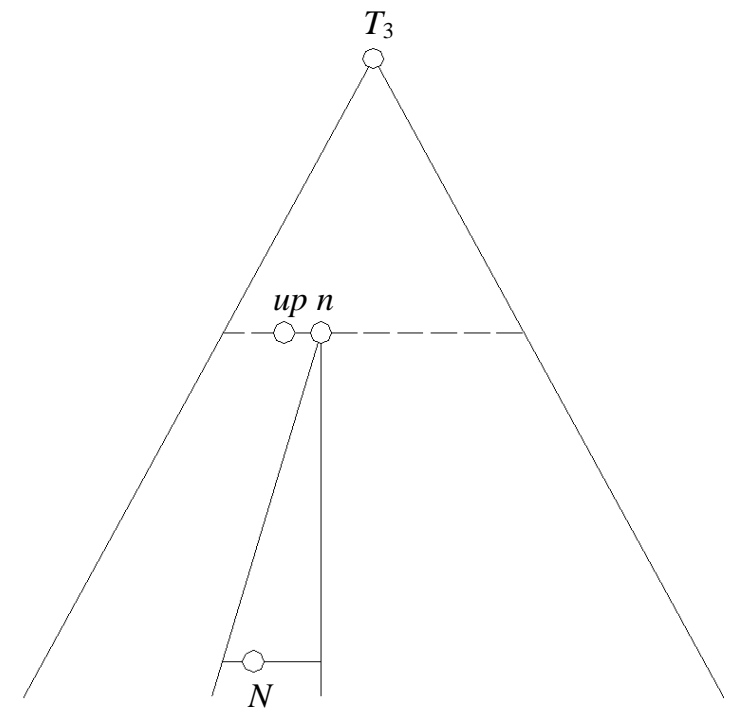

Fig. 11: Relations among $n, N$ and $u p$ 
Table 2: Summarized cases from Corollaries 6 and 7

\begin{tabular}{lllll}
\hline$p$ & $q$ & $J$ & $N$ \\
\hline $2^{\alpha}+1<p \leq 2^{\alpha+1}-1$ & $q=2^{\alpha} u-1$ & $2^{\alpha-1} \leq 2^{\alpha-1}+2^{\alpha}-\frac{p+1}{2}<2^{\alpha}-1$ & $N=N_{\left(\alpha, 2^{\alpha}+2^{\alpha-1}-\frac{p+1}{2}\right)}^{u-2}$ & $N \in r\left(T_{u p-2}\right)$ \\
& $q=2^{\alpha} u-1$ & $0<2^{\alpha-1}-2^{\alpha}+\frac{p-1}{2} \leq 2^{\alpha-1}-1$ & $N=N_{\left(\alpha, \frac{p-1}{2}-2^{\alpha-1}\right)}^{u p+2}$ & $N \in l\left(T_{u p+2)}\right.$ \\
\hline
\end{tabular}

Table 3: Ten factorized samples

\begin{tabular}{ll}
\hline Odd Integers & Factorizaion \\
\hline 34639739 & $8191 \times 4229$ \\
1159847279 & $131071 \times 8849$ \\
10581684521 & $524287 \times 20183$ \\
60782931320919664123 & $59649589127497217 \times 1019$ \\
10263855667940024299 & $1256132134125569 \times 8171$ \\
115271397873601774304441 & $2305843009213693951 \times 49991$ \\
174538042279885450969073 & $2663848877152141313 \times 65521$ \\
944515611538471874461691 & $3603109844542291969 \times 262139$ \\
2732669846011417649053579 & $167988556341760475137 \times 16267$ \\
5057672949897463733694209 & $18446744073709551617 \times 274177$ \\
\hline
\end{tabular}

\section{Algorithm and Numerical Experiments}

\section{Algorithm}

Theorems 4 and 5 provide an approach to factorize rapidly a composite odd integer $N=p q$ if $q$ is in the form $q=2^{\alpha} u \pm 1$ and $p$ satisfies $1<p \leq 2^{\alpha} \pm 1$ or $2^{\alpha}+1<p \leq 2^{\alpha}$ $+1-1$. This section presents a factoring algorithm. The whole procedure includes two subroutines and a main routine as follows.

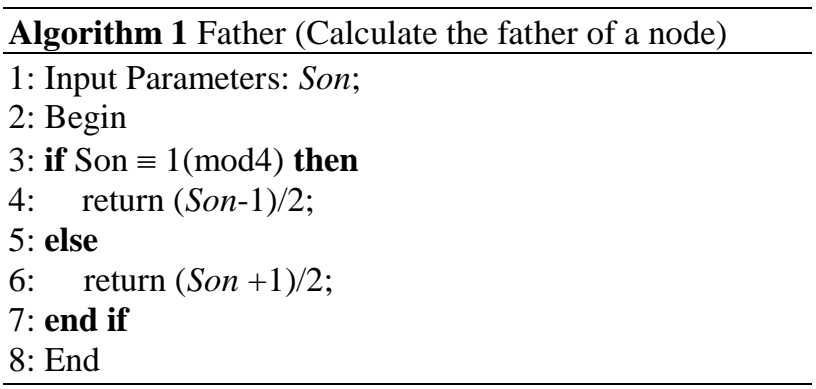

The main routine shows, it requires at most $3\left\lfloor\log _{2} N\right\rfloor$ +1 searching steps to factorize $N$. Since at each searching step, it needs $O\left(\left(\log _{2} N\right)^{3}\right)$ bit operations to compute the GCD, it knows that the total computation can be completed in $O\left(\left(\log _{2} N\right)^{4}\right)$ bit operations.

\section{Numerical Experiments with Maple 15}

With the algorithm, programs in Maple are designed as list in the appendix. With the programs, ten odd integers are factorized in milliseconds in Maple. The ten numbers are list in Table 3. The biggest one is a 25 decimal-digit number 5057672949897463733694209.

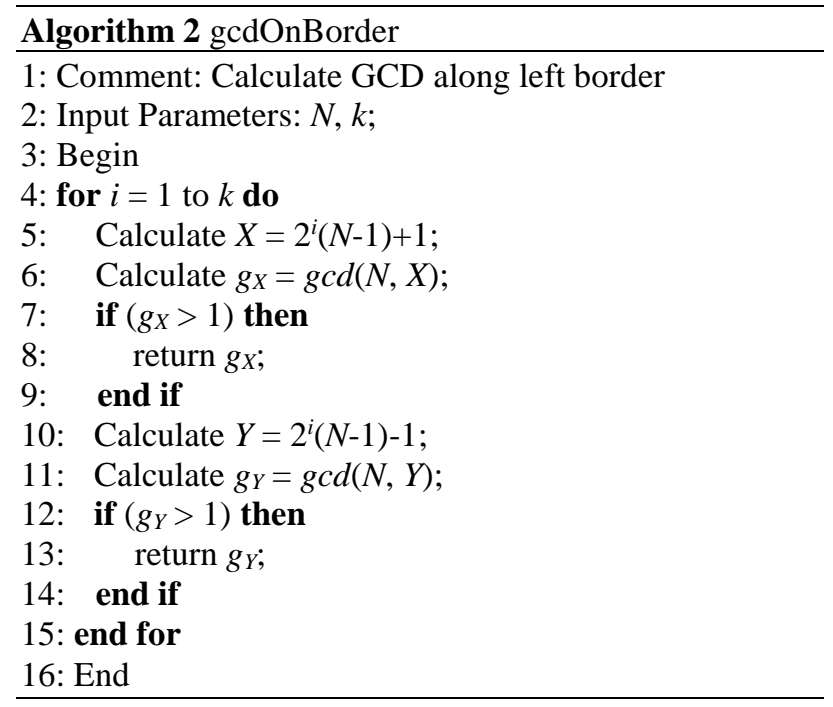

\section{Conclusion and Future Work}

Looking through the theorems and corollaries proved in previous sections, one can easily know that, for an odd composite integer $N=p q$ with $q$ being in the form of $2^{\alpha} u$ \pm 1 and $\mathrm{p}$ satisfying $1<p \leq 2^{\alpha} \pm 1$ or $2^{\alpha}+1<p \pm 2^{\alpha+1}-1$, it is easy to factorize $N$ with the help of the valuated binary tree $T_{N}$. Actually, the factorization can be completed by just tracing and finding in $T_{N}$ the GCD between $N$ and $N$ 's ancestors or between $N$ and the leftmost path $P_{0}^{N}$ as well as the left side-path $P_{L}^{N}$. Since there are a lot of odd positive integers that fit the conditions, this paper surely solves part of the problem on factoring big odd integers.

Meanwhile, readers can see from the list of bibliographies and their related references that, the tree method is in deed a valid method to study integers. This 
leads to the future work. Hope more gougers join the study and solve the hard problem of integer factorization.

\section{Acknowledgement}

The research is supported by the Open Project Program of the State Key Lab of CAD\&CG（Grant No. A2002) and by Foshan University and Foshan Bureau of Science and Technology under project that constructs Guangdong Engineering Center of Information Security for Intelligent Manufacturing System.

\section{Author's Contributions}

Prof. Xingbo WANG contributes $95 \%$ of the work in this paper, including discovering and proving the corollaries and theorems as well as designing the algorithm. Mr. Junjian ZHONG contributes 5\% of the work, mainly programs and does numerical experiments.

\section{Ethics}

The authors declare that there is no conflict of interests regarding the publication of this article.

\section{References}

Calik, P., Yilgora P., Ayhanb P. and Demir A.S., 2004. Oxygen transfer effects on recombinant benzaldehydelyase production. Chem. Eng. Sci., 59: 5075-5083. DOI: 10.1016/j.ces.2004.07.070

Phulachand, K.S., 2016. An overview of cryptography. Innovat. IT, 3: 8-11.

Sarnaik, S., Gadekar D. and Gaikwad U., 2016. An overview to integer factorization and RSA in cryptography. Int. J. Adv. Res. Eng. Thechnol., 2: 21-26.

Wang, X., 2016a. Valuated binary tree: A new approach in study of integers. Int. J. Scientific Innovat. Math. Res., 4: 63-67. DOI: 10.20431/2347-3142.0403008

Wang X., 2016b. Amusing properties of odd numbers derived from valuated binary tree. IOSR J. Math., 12: $53-57$

Wang, X., 2017a. Two more symmetric properties of odd numbers. IOSR J. Math., 13: 37-40. DOI: $10.9790 / 5728-1303023740$

Wang, X., 2017b. Genetic traits of odd numbers with applications in factorization of integers. Global $\mathrm{J}$. Pure Applied Math., 13: 493-517.

Wang, X., 2018. T3 tree and its traits in understanding integers. Adv. Pure Math., 8: 494-507.

DOI: 10.4236/apm.2018.85028

Wang, X., 2019. Brief summary of frequently- used properties of the floor function: updated 2018. IOSR J. Math., 15: 30-33.
Wang, X. and Guo H., 2019. Some divisibility traits on valuated binary trees. Int. J. Applied Phys. Math., 9: 1-15. DOI: 10.17706/ijapm.2019.9.4.173-181

Yan, S.Y., 2013. Computer Number Theory and Modern Cryptography. 1st Edn., John Wiley and Sons, Singapore, ISBN-10: 1118188586, pp: 432.

\section{Appendix: Maple Programs and Running Results}

\#Subroutine Father: find the father of a node

Father := proc $(\mathrm{S})$

local X, r;

$\mathrm{r}:=\operatorname{modp}(\mathrm{S}, 4)$;

if $r=1$ then $X:=(1 / 2) * S+1 / 2$

else $\mathrm{X}:=(1 / 2) * \mathrm{~S}-1 / 2$ end if;

end proc

\#Subroutine gcdOnBorder

$\operatorname{gcdOnBorder}:=\operatorname{proc}(\mathrm{N}, \mathrm{k})$

local X, g, i;

for $\mathrm{i}$ to $\mathrm{k}$ do

$$
\begin{aligned}
& X=2^{i} *(\mathrm{~N}-1)+1 ; \\
& \mathrm{g}:=\operatorname{gcd}(\mathrm{N}, \mathrm{X}) ; \\
& \text { if } 1<\mathrm{g} \text { then break end if; } \\
& X=2^{i} *(\mathrm{~N}-1)-1 ; \\
& \mathrm{g}:=\operatorname{gcd}(\mathrm{N}, \mathrm{X}) ; \\
& \text { if } 1<g \text { then break end if; }
\end{aligned}
$$

end do;

end proc

\# Main routine

doit:=proc $(\mathrm{N})$

local k,F,i,g;

$k:=$ floor $((\log (\mathrm{N})) /(\log (2)))+1$;

$\mathrm{g}:=\operatorname{gcdOnBorder}(\mathrm{N}, \mathrm{k})$ :

if $g>1$ then $\operatorname{return}(\mathrm{g})$ : fi;

$\mathrm{F}:=\mathrm{N}$;

for $\mathrm{i}$ from 1 to $\mathrm{k}$ do

$\mathrm{F}:=$ Father(F);

$\mathrm{g}:=\operatorname{gcd}(\mathrm{N}, \mathrm{F})$;

if $g>1$ then $\operatorname{return}(\mathrm{g})$ : fi;

$\mathrm{g}:=\operatorname{gcd}(\mathrm{N}, \mathrm{F}-2)$;

if $g>1$ then $\operatorname{return}(\mathrm{g})$ : fi;

$\mathrm{g}:=\operatorname{gcd}(\mathrm{N}, \mathrm{F}+2)$;

od;

if $g>1$ then $\operatorname{return}(\mathrm{g})$ : fi;

end proc

\#tested numbers

ob := Array(1 .. 10, [34639739, 1159847279 ,

10581684521, 10263855667940024299,

60782931320919664123 ,

115271397873601774304441 ,

174538042279885450969073 ,

944515611538471874461691,

2732669846011417649053579 ,

5057672949897463733694209]); 
Xingbo Wang and Junjian Zhong / Journal of Mathematics and Statistics 2020, Volume 16: 24.34 DOI: $10.3844 /$ jmssp.2020.24.34

\section{\# test commands \\ for $\mathrm{i}$ to $10 \mathrm{do}$ \\ d1 := doit(ob[i]); \\ $\mathrm{d} 2:=\mathrm{ob}[\mathrm{i}] / \mathrm{d} 1$; \\ $\operatorname{lprint}(\mathrm{ob}[\mathrm{i}], \mathrm{d} 1, \mathrm{~d} 2)$ \\ end do; \\ \# Test results}

$34639739,4229,8191$

$1159847279,8849,131071$

$10581684521,20183,524287$

$10263855667940024299,8171,1256132134125569$

$60782931320919664123,1019,59649589127497217$

115271397873601774304441, 49991, 2305843009213693951

$174538042279885450969073,65521,2663848877152141313$

944515611538471874461691，262139, 3603109844542291969

$2732669846011417649053579,16267,167988556341760475137$

5057672949897463733694209, 274177, 18446744073709551617
4229

8849

131071

20183

524287

8171

1256132134125569

1019 59649589127497217

49991

2305843009213693951

65521

2663848877152141313

262139

3603109844542291969

16267

167988556341760475137

$$
274177
$$

18446744073709551617 